\title{
Amyotrophic Lateral Sclerosis: Drug Therapy from the Bench to the Bedside
}

\author{
Summer Bell Gibson, M.D. ${ }^{1}$ Mark B. Bromberg, M.D., Ph.D. ${ }^{1}$ \\ ${ }^{1}$ Department of Neurology, University of Utah School of Medicine, Salt \\ Lake City, Utah \\ Semin Neurol 2012;32:173-178. \\ Address for correspondence and reprint requests Summer Gibson, \\ MD, Department of Neurology, University of Utah, Clinical \\ Neurosciences Center, 175 N Medical Drive East, Salt Lake City, Utah \\ 84132 (e-mail: summer.gibson@hsc.utah.edu).
}

\author{
Abstract \\ Keywords \\ - amyotrophic lateral \\ sclerosis \\ - ALS \\ - Lou Gehrig's disease \\ - treatments and \\ investigational drugs
}

Amyotrophic lateral sclerosis (ALS) is an unrelenting progressive neurodegenerative disease causing progressive weakness, ultimately leading to death. Despite aggressive research, the pathways leading to neuronal death are incompletely understood. Riluzole is the only drug clinically proven to enhance survival of ALS patients, but its mechanism of action is not clearly understood. In this article, the proposed pathophysiology of ALS is reviewed including glutamate excitotoxicity, oxidative stress, mitochondrial dysfunction, autoimmune mechanisms, protein aggregation, SOD1 accumulation, and neuronal death. Based on these mechanisms, past major ALS drug studies will be reviewed as well as promising current ALS drug studies, focusing on the advancement of these studies from the bench to the patient's bedside.
Amyotrophic lateral sclerosis (ALS) is a neurodegenerative disease characterized by progressive muscular atrophy and weakness resulting from loss of both upper and lower motor neurons. It was first clinically and pathologically described by Charcot in $1874 .^{1}$ Amyotrophic lateral sclerosis gained notoriety after the New York Yankee baseball player, Lou Gehrig, was diagnosed in 1939; to this day, it is commonly referred to as "Lou Gehrig's disease" in the United States. The disease generally progresses rapidly and is inevitably fatal. The cause of death is relatively uniform-typically due to respiratory failure. $^{2,3}$ The incidence ranges from 1.5 to 2.5 per 100,000 per year, with a lifetime risk of $\sim 1: 400 .^{4}$ The mean age of onset is $\sim 60$ years, with a male predominance of $1.3: 1 .^{5}$ The median survival is 2 to 4 years from symptom onset, although a small percentage live longer than 10 years. The differential diagnosis is small and misdiagnosis is estimated to be less than $10 \%{ }^{6,7}$

Familial ALS is clinically indistinguishable from sporadic ALS. Familial ALS is generally defined as the history or presence of ALS in one or more $1^{\text {st }}$ - or $2^{\text {nd }}$-degree family members of a person with ALS. ${ }^{8,9}$ The rate of familial ALS is 5 to $10 \% .{ }^{9,10}$ In 1993 , a superoxide dismutase (SOD1) mutation was discovered that can lead to ALS. Despite this advance, it was nearly a decade before the next gene mutation was discovered. There has recently been a rapid expansion in the number of recognized ALS mutations, with 10 different ALS mutations identified. ${ }^{10,11}$ It is estimated that the most common ALS genes, SOD1, TDP-43, and FUS mutations and the C9orf72 hexanucleotide repeat, account for $65 \%$ of familial ALS cases in the United States, although the percentage of ALS linked to these genes varies based on geographical region. ${ }^{12,13}$ For some of the genes, ALS is not the only neurodegenerative phenotype expressed. For example, the C9orf72 hexanucleotide repeat, is also a common cause of frontotemporal dementia (FTD), sometimes in combination with ALS, and sometimes in isolation with either ALS or FTD. ${ }^{12}$

There are currently $>50$ actively enrolling clinical trials for ALS listed on clinicaltrials.gov, with several times that number completed. Most drugs for ALS come through a traditional route of identifying a target and then screening for compounds that modify the activity of the target, then optimizing hits within the identified drug family to help select the ideal compound. However, drugs are increasingly coming from large screening efforts that examine compounds without known targets. This helps to identify potential drug treatments with mechanisms already known or thought to be related to ALS, but also identify potential drug treatments that cause reconsideration of the disease pathway. One of the first nontraditional screens for ALS and other selected neurologic diseases was the large community screening effort by
Issue Theme Neuromuscular Therapy from Bench to Bedside; Guest Editor, A. Gordon Smith, MD
Copyright (c) 2012 by Thieme Medical Publishers, Inc., 333 Seventh Avenue, New York, NY 10001, USA. Tel: +1(212) 584-4662.
DOI http://dx.doi.org/ $10.1055 / \mathrm{s}-0032-1329193$. ISSN $0271-8235$. 
the National Institute of Neurological Disorders and Stroke (NINDS) in the early 2000s. ${ }^{14}$ About 1,000 compounds were screened in a variety of biologic assays, the majority of which were already in use for nonneurologic diseases, and several had positive results. Here we will review proposed ALS disease mechanisms followed by historical and upcoming drug studies.

\section{Disease Mechanisms}

Although the exact cause of ALS is unknown, there are many theories that may represent a cascade of pathologic changes. An early and leading theory is that glutamate, the central nervous system's most abundant excitatory neurotransmitter, causes neuron death when it is elevated, leading to the development of ALS. This was supported by a combination of findings in ALS patients including elevated glutamate levels found in fasting serum and cerebrospinal (CSF), ${ }^{15,16}$ a deficiency of leukocyte glutamate dehydrogenase, ${ }^{17}$ and defects in the glutamate transport system that lead to decreased clearance of extracellular glutamate. ${ }^{18}$

Oxidative stress is another prominent area of interest. The potential importance of antioxidant dysfunction was boosted with the discovery that SOD1 mutations cause familial ALS. SOD1 is a powerful antioxidant enzyme that catalyzes the dismutation of the highly reactive superoxide free radical generated in mitochondria, thus keeping it from harming cell structures. ${ }^{19}$ However, it is now believed that SOD1 mutations cause disease by a gain of function related to overexpression of the mutant SOD1 enzyme, ${ }^{20}$ leaving the role of antioxidant function in ALS less clear.

There is evidence supporting an autoimmune mechanism in ALS. ${ }^{21}$ It is hypothesized that the immune system targets the motor nerve terminal leading to a series of changes that alter calcium homeostasis. ${ }^{22}$ The disruption of calcium homeostasis may trigger neuronal cell death through apoptotic pathways. ${ }^{23}$ Additionally, the ALS SOD1 mouse model demonstrates increased inflammatory factors throughout its lifespan, including during presymptomatic stages. ${ }^{24}$ Macrophages, which play a key role in neuroinflammation, have been found at increased levels in spinal cord tissue of sporadic and familial ALS patients as well as in the ALS SOD1 mouse model. ${ }^{25}$

The role of abnormal protein aggregation has been gaining support in neurodegenerative diseases including ALS. Proteins that may misfold in ALS include SOD1, TDP-43, and FUS. Cellto-cell propagation of misfolded proteins may involve a prionlike phenomena. ${ }^{26}$ This basic finding may underlie the clinical observation of somatotopic spread of weakness in most ALS patients. Misfolded SOD1 proteins have been shown to induce the misfolding of normal wild type SOD1 in cell culture. ${ }^{27}$ This is supported by pathologic evidence that shows ALS begins focally and then spreads to neighboring neurons. ${ }^{28}$

To facilitate the study of these hypotheses and to explore new treatments, animal models based on genetic mutations have been developed. SOD1 gene mutations were discovered before other ALS gene mutations and remain the most used and best described animal models. However, there is growing concern that the SOD1animal model may not represent ALS as a whole. SOD1 mutations only cause $\sim 2 \%$ of all ALS, ${ }^{19}$ and thus the mechanisms may be distinct from other forms of ALS. ${ }^{29,30}$ To address this concern, particularly with drugs that target the SOD1 mutation, some ALS drug studies only include patients with known SOD1 mutations.

\section{Stem Cell Therapy}

ALS is an area of intense interest for stem cell transplant research. Unfortunately, many ALS patients have fallen prey to false promises and stem cell scams, both in the United States and abroad. $^{31,32}$ Stem cell studies have yielded positive results in both in vitro and ALS animal models using a variety of different cell types. Mesenchymal stem cells (bone marrow derived) and neural progenitor cells (spinal cord-derived) are the two cell types with the most evidence for use in ALS. Both mesenchymal and neural progenitor cells have supporting data from SOD1 animal model studies, demonstrating improved survival, when compared with control animals. ${ }^{33,34}$ Mesenchymal stem cells have the advantage of autologous implantation, thus reducing rejection issues and the need for immunosuppressant therapy. However, recent studies show that mesenchymal cells isolated from ALS patients have reduced pluripotency and trophic factors and thus suggest a reduced potential for autologous mesenchymal transplants in ALS patients. ${ }^{35}$

\section{Antiglutamate Drugs}

Riluzole was developed in the 1950s as a centrally acting muscle relaxant and later investigated as an antiseizure and a neuroprotective agent. ${ }^{36}$ The exact mechanism of action of riluzole is unknown, but it has multiple properties, including inhibition of sodium, calcium, potassium, and glutamate currents. ${ }^{36}$ In preclinical studies, riluzole was found to modulate the transmission of glutamate in hippocampal slices. ${ }^{37}$ After clinical studies in ALS, riluzole was approved by the Food and Drug Administration (FDA) in 1995 for treatment. The clinical benefits are modest, extending ventilator-free survival by $\sim 3$ months, ${ }^{38}$ but it remains the only FDAapproved disease-modifying drug for ALS.

Other antiglutamate drugs have been evaluated in clinical trials. Most are antiseizure medications that have antiglutamate properties, and include topiramate, gabapentin, and lamotrigine, but none have demonstrated survival benefit. Topiramate, a sugar derivative, has four main properties: inhibition of the enzyme voltage-dependent sodium channels, inhibition of carbonic anhydrase, enhancement of some GABA-A receptors, and antagonism of glutamate receptors. ${ }^{39}$ Initial topiramate studies with organotypic spinal cord culture were promising; however, it failed to improve survival in the ALS SOD 1 mouse model. ${ }^{40}$ This was followed by a doubleblind placebo-controlled, multicenter randomized clinical trial with 296 ALS patients. ${ }^{41}$ In this study, those treated with topiramate actually had a faster decline in upper-extremity strength than those treated with placebo, and it failed to result in increased survival, or change in the decline in 
forced vital capacity (FVC) or the ALS Functional Rating Scale (ALSFRS). Gabapentin primarily works by inhibiting voltagegated calcium channels, but at high doses reduces glutamate synthesis. ${ }^{42}$ When phase II and phase III results were combined, there was a significantly increased rate of decline in FVC in the gabapentin-treated group, and the studies failed to result in any improvement in survival or change in the rate of decline in ALSFRS or timed walk. ${ }^{43}$ Lamotrigine is a sodium channel blocker that also inhibits the release of glutamate and aspartate. ${ }^{21,26}$ Lamotrogine has been studied in two doubleblind placebo controlled trials, the first at low dose (100 mg daily) and the second at a moderate dose (300 mg daily), but both studies failed to show any improvement in ambulation, bulbar symptoms, or ALSFRS. ${ }^{44,45}$ Other drugs with antiglutamate proprieties, including dextromethorphan and memantine, failed to show improvement in survival. ${ }^{46,47}$

Despite these multiple negative studies, several antiglutamate drugs show promise and are currently in development stages. Talampanel (LY300164), a benzodiazepine that is a noncompetitive AMPA antagonist with antiglutamate properties, has completed a phase II drug trial with 59 ALS patients. ${ }^{48}$ The results were mixed, but had promising aspects; the decline in muscle strength was slowed by $15 \%$ and the decline in the ALSFRS was slowed by $30 \%$ in the talampanel-treated group; nevertheless, neither measure reached statistical significance, and there was no survival benefit. In a recent SOD1 ALS mouse study with talampanel, motor neuron calcium levels were reduced, but only when given presymptomatically. ${ }^{49}$ Ceftriaxone, a third-generation cephalosporin, which likely modifies glutamate by altering the glutamate transport protein, ${ }^{50}$ is currently in a phase III trial, with 600 ALS participants. ${ }^{51}$ Glutamate carboxypeptidase II (NAALADase) has been proposed because of its dual antiglutamate mechanism, by directly decreasing production of glutamate and indirectly increasing the breakdown of glutamate in the central nervous system. ${ }^{52}$ Additionally, glutamate carboxypeptidase II has been shown to reduce neuropathologic changes in the ALS SOD1 mouse model. ${ }^{52}$

\section{Antioxidant/Mitochondrial Preservation Drugs}

The first study of an antioxidant agent (vitamin E) for ALS was published in $1940 .{ }^{53}$ This study had several critical design flaws and remains an example of the need for controls and blinding. Included in the multiple vitamin E responders was Lou Gehrig himself, who reportedly "improved" with treatment. ${ }^{53,54}$ Despite continuing his treatment with high doses of oral and injected vitamin $\mathrm{E}$, he passed away one year later. ${ }^{54}$ Interest in vitamin $\mathrm{E}$ was rekindled due to delayed disease onset in the ALS SOD1 mouse model. ${ }^{55}$ Two randomized controlled double-blind clinical studies of vitamin E or placebo in combination with riluzole have now been completed. One used 600 IU daily and the other 5,000 mg daily. Neither showed benefit on survival or functional status when vitamin E was added to riluzole. ${ }^{56,57}$ However, the issue remains open, as there have been recent studies suggesting a decreased ALS risk among long-term vitamin E users. ${ }^{58}$
Other antioxidant agents and other drugs targeted to mitochondrial function have also been investigated. $\mathrm{N}$-acetylcysteine, an over-the-counter antioxidant, significantly prolonged survival and delayed motor symptom onset when given presymptomatically in the ALS SOD1 mouse model. ${ }^{59}$ However, a clinical ALS trial with $\mathrm{N}$-acetylcysteine failed to produce significant differences in survival or change in decline of motor symptoms. ${ }^{60}$ Creatine, which has neuroprotective effects, also had positive animal data, but the human ALS studies failed to show significant differences in survival, ALSFRS-revised (ALSFRS-R), or FVC in those treated with 5 to $10 \mathrm{~g}$ of creatine. ${ }^{61}$ The antidiabetes type II drug, metformin, with antioxidant and antiinflammatory properties, showed no benefit in male ALS SOD1 mice and accelerated disease progression in the female mice. ${ }^{62}$ There have been many other small studies with antioxidants, such as selegiline and melatonin, and though these failed to produce positive results they are generally too small to draw significant conclusions. ${ }^{63,64}$

Despite multiple negative antioxidant drug trials, one agent remains promising. Dexpramipexole, the $\mathrm{R}+$ enantiomer of pramipexole, has antioxidant effects, in part by targeting preservation of mitochondria function by reducing apoptosis. ${ }^{65}$ The S-enantiomer, pramipexole is currently used for Parkinson's disease and restless leg syndrome. The pure $\mathrm{R}+$ form has less dopaminergic receptor affinity and thus reduces many of the dose-limiting dopaminergic side-effects. ${ }^{65}$ Dexpramipexole, is currently in phase III studies ${ }^{66}$ based on a promising two-part phase II trial, which showed a dose-dependent trend toward a slower decline in the ALSFRS$\mathrm{R}$ and significant difference in decline of both mortality and ALSFRS-R. ${ }^{67}$

\section{Immunosuppressive Drugs and Procedures}

Multiple immunosuppressive drugs have been studied, including corticosteroids, plasmapheresis, intravenous immunoglobulin, cyclophosphamide, and cyclosporine, all of which failed to alter disease progression. ${ }^{68-72}$ Minocycline is a tetracycline antibiotic that decreases inflammation by inhibiting microglial activation. ${ }^{73}$ SOD1 animal studies were optimistic, showing delayed disease onset, prolonged survival, and decreased motor neuron loss when given to presymptomatic animals. ${ }^{73}$ In phase $\mathrm{I} / \mathrm{II}$ trials there were no major safety issues. ${ }^{74}$ In a phase III randomized placebo-controlled trial, those treated with minocycline had a significantly greater decline in the ALSFRS-R score. ${ }^{75}$ There was also a nonsignificant trend toward a faster decline in breathing function and muscle strength as well as mortality. These results were supported by experiments in the ALS SOD1 mouse model that showed when minocycline was given late in the symptomatic phase it no longer had a neuroprotective effect, but caused an increased inflammatory response. $^{76}$

A new investigational agent, NP001, targets another mechanism of neuroinflammation, by regulating macrophage activation and potentially returning macrophages back to their neuroprotective state. ${ }^{77}$ In the phase I study, patients were 
given four doses of ascending quantity and ALS progressionassociated biomarkers were measured. ${ }^{77}$ The drug was determined to be safe and tolerable at doses given. Additionally, ALS progression biomarkers levels had a statistically significant dose-dependent decrease to each of the NP001 treatments. NP001 is currently in a phase II study. ${ }^{78}$

\section{Muscle-Maintenance Drugs}

Another proposed target for the drug treatment of ALS is at the muscle itself. CK-2017357 activates skeletal muscle by causing it to be more sensitive to calcium. ${ }^{79}$ By lowering the muscle sensitivity to calcium it is theorized to increase the force produced by the muscle stimulus. ${ }^{79}$ A double-blind randomized placebo-controlled phase II trial of CK-2017357 has been completed. ${ }^{80}$ Part A of the phase II trial included patients not taking riluzole, and part $B$ included patients taking riluzole but at a reduced dose of $50 \mathrm{mg}$ daily (instead of $50 \mathrm{mg}$ twice a day). The treated patients had a dose-dependent improvement in maximum ventilation and handgrip endurance that trended toward significance.

\section{SOD1 Specific Treatments}

ISIS-SOD1rx is an antisense oligonucleotide that targets and reduces the synthesis of SOD1. It has been shown to prolong survival in symptomatic ALS SOD1 rats. ${ }^{81}$ Phase I trials to determine safety of ISIS-SOD1rx in familial ALS patients with a confirmed SOD1 mutation have been completed and the agent appears safe. ${ }^{82,83}$

Arimoclomol and pyrimethamine, both of which are currently in clinical trial, are also being tested in patients with SOD1 mutations. Arimoclomol, which is in phase II/III, has been shown to increase survival and improve motor function in the ALS SOD1 mouse model. ${ }^{84,85}$ Arimoclomol is believed to protect motor neurons from cell death by amplifying the cytoprotective heat shock response in times of stress. ${ }^{84}$ Pyrimethamine, an antimalarial and toxoplasmosis drug, has been found to reduce in vitro levels of SOD1 in mice and humans. ${ }^{86,87}$ It was identified as a potential SOD1 lowering agent through a high-throughput screen, and a phase I/II study is underway. ${ }^{88}$

\section{Stem Cells}

The first stem cell trial for ALS surgically implanted autologous mesenchymal cells into the dorsal spinal cord of 19 ALS patients. ${ }^{89}$ This study has now completed a two-part phase I trial with long-term follow-up, and the treatment appears to be reasonably safe. The second mesenchymal safety study included both multiple sclerosis (MS) and ALS patients. ${ }^{90}$ Nineteen ALS patients had autologous enhanced mesenchymal cells injected intrathecally and intravenously. Patients were followed from 6 to 18 months and the procedure was felt to be reasonably safe. The first human controlled trial with neural stem cells is currently underway. ${ }^{91}$ Part 1 of the phase 1 study demonstrated that lumbar intraspinal injections of neural stem cells (spinal cord derived cells) was safe in 12 patients. ${ }^{91}$ Although the study was not intended nor powered for efficacy, one subject had striking improvement in their ALSFRS-R score. Part 2 of phase 1 is underway with intraspinal injections in the cervical cord, with the goal of preferentially protecting respiratory motor neurons. ${ }^{91}$

\section{Conclusion}

The race to understand and treat ALS is on. Despite aggressive research, riluzole remains the only FDA-approved pharmacological therapy for ALS. The myriad mechanisms of ALS pathophysiology discussed, including glutamate excitotoxicity, the role of antioxidants, mitochondrial dysfunction, autoimmune components, and protein aggregation, suggest that ALS is a complex disease for which we still know remarkably little. Although there have been positive results in pharmacologic targeting of all of the mechanisms discussed in this review, no agent has been developed that significantly alters the natural history. While curative drugs have been discovered in the past for other diseases without a complete understanding of the pathophysiology or all of the relevant targets, it is unlikely that major breakthroughs in ALS treatment will come without a more complete understanding of the true disease mechanism.

We eagerly await the phase III results of the antiglutamate drug ceftriaxone, and the mitochondria preservation drug, dexpramipexole. Given the multiple human studies that failed to confirm findings from animal studies, we are cautious about placing too much emphasis on the results from animal studies, particularly when completed in the asymptomatic phase.

\section{References}

1 Charcot JM. De la sclérose latérale amyotrophique. Prog Med 1874;2:325-455

2 Gil J, Funalot B, Verschueren A, et al. Causes of death amongst French patients with amyotrophic lateral sclerosis: a prospective study. Eur J Neurol 2008;15(11):1245-1251

3 Spataro R, Lo Re M, Piccoli T, Piccoli F, La Bella V. Causes and place of death in Italian patients with amyotrophic lateral sclerosis. Acta Neurol Scand 2010;122(3):217-223

4 Hardiman O, van den Berg LH, Kiernan MC. Clinical diagnosis and management of amyotrophic lateral sclerosis. Nat Rev Neurol 2011;7(11):639-649

5 McCombe PA, Henderson RD. Effects of gender in amyotrophic lateral sclerosis. Gend Med 2010;7(6):557-570

6 Belsh JM, Schiffman PL. Misdiagnosis in patients with amyotrophic lateral sclerosis. Arch Intern Med 1990;150(11):2301-2305

7 Chiò A. ISIS Survey: an international study on the diagnostic process and its implications in amyotrophic lateral sclerosis. J Neurol 1999;246(Suppl 3):III1-III5

8 Byrne S, Elamin M, Bede P, Hardiman O. Absence of consensus in diagnostic criteria for familial neurodegenerative diseases. J Neurol Neurosurg Psychiatry 2012;83(4):365-367

9 Byrne S, Walsh C, Lynch C, et al. Rate of familial amyotrophic lateral sclerosis: a systematic review and meta-analysis. J Neurol Neurosurg Psychiatry 2011;82(6):623-627

10 Siddique T, Ajroud-Driss S. Familial amyotrophic lateral sclerosis, a historical perspective. Acta Myol 2011;30(2):117-120 
11 Renton AE, Majounie E, Waite A, et al; ITALSGEN Consortium. A hexanucleotide repeat expansion in C9ORF72 is the cause of chromosome 9p21-linked ALS-FTD. Neuron 2011;72(2):257-268

12 Majounie E, Renton AE, Mok K, et al; Chromosome 9-ALS/FTD Consortium; French research network on FTLD/FTLD/ALS; ITALSGEN Consortium. Frequency of the C9orf72 hexanucleotide repeat expansion in patients with amyotrophic lateral sclerosis and frontotemporal dementia: a cross-sectional study. Lancet Neurol 2012;11(4):323-330

13 Andersen PM, Al-Chalabi A. Clinical genetics of amyotrophic lateral sclerosis: what do we really know? Nat Rev Neurol 2011;7(11):603-615

14 Abbott A. Neurologists strike gold in drug screen effort. Nature 2002;417(6885):109

15 Plaitakis A, Caroscio JT. Abnormal glutamate metabolism in amyotrophic lateral sclerosis. Ann Neurol 1987;22(5):575-579

16 Rothstein JD, Tsai G, Kuncl RW, et al. Abnormal excitatory amino acid metabolism in amyotrophic lateral sclerosis. Ann Neurol 1990;28(1):18-25

17 Hugon J, Tabaraud F, Rigaud M, Vallat JM, Dumas M. Glutamate dehydrogenase and aspartate aminotransferase in leukocytes of patients with motor neuron disease. Neurology 1989;39(7): 956-958

18 Rothstein JD, Martin LJ, Kuncl RW. Decreased glutamate transport by the brain and spinal cord in amyotrophic lateral sclerosis. N Engl J Med 1992;326(22):1464-1468

19 Rosen DR. Mutations in $\mathrm{Cu} / \mathrm{Zn}$ superoxide dismutase gene are associated with familial amyotrophic lateral sclerosis. Nature 1993;364(6435):362

20 Shibata N. Transgenic mouse model for familial amyotrophic lateral sclerosis with superoxide dismutase-1 mutation. Neuropathology 2001;21(1):82-92

21 Pagani MR, Gonzalez LE, Uchitel OD. Autoimmunity in amyotrophic lateral sclerosis: past and present. Neurol Res Int 2011;2011:497080

22 Pagani MR, Reisin RC, Uchitel OD. Calcium signaling pathways mediating synaptic potentiation triggered by amyotrophic lateral sclerosis IgG in motor nerve terminals. J Neurosci 2006;26 (10):2661-2672

23 Orrenius S, Zhivotovsky B, Nicotera P. Regulation of cell death: the calcium-apoptosis link. Nat Rev Mol Cell Biol 2003;4(7):552-565

24 Alexianu ME, Kozovska M, Appel SH. Immune reactivity in a mouse model of familial ALS correlates with disease progression. Neurology 2001;57(7):1282-1289

25 Henkel JS, Engelhardt JI, Siklós L, et al. Presence of dendritic cells, MCP-1, and activated microglia/macrophages in amyotrophic lateral sclerosis spinal cord tissue. Ann Neurol 2004;55(2): 221-235

26 Polymenidou M, Cleveland DW. The seeds of neurodegeneration: prion-like spreading in ALS. Cell 2011;147(3):498-508

27 Grad LI, Guest WC, Yanai A, et al. Intermolecular transmission of superoxide dismutase 1 misfolding in living cells. Proc Natl Acad Sci U S A 2011;108(39):16398-16403

28 Ravits J, Laurie P, Fan Y, Moore DH. Implications of ALS focality: rostral-caudal distribution of lower motor neuron loss postmortem. Neurology 2007;68(19):1576-1582

29 Benatar M. Lost in translation: treatment trials in the SOD1 mouse and in human ALS. Neurobiol Dis 2007;26(1):1-13

30 Scott S, Kranz JE, Cole J, et al. Design, power, and interpretation of studies in the standard murine model of ALS. Amyotroph Lateral Scler 2008;9(1):4-15

31 The darker side of stem cells. Nature 2012;483(7387):5

32 Cyranoski D. China's stem-cell rules go unheeded. Nature 2012;484(7393):149-150

33 Uccelli A, Milanese M, Principato MC, et al. Intravenous mesenchymal stem cells improve survival and motor function in experimental amyotrophic lateral sclerosis. Mol Med 2012;18(1): 794-804
34 Xu L, Yan J, Chen D, et al. Human neural stem cell grafts ameliorate motor neuron disease in SOD-1 transgenic rats. Transplantation 2006;82(7):865-875

35 Koh SH, Baik W, Noh MY, et al. The functional deficiency of bone marrow mesenchymal stromal cells in ALS patients is proportional to disease progression rate. Exp Neurol 2012;233(1):472-480

36 Bellingham MC. A review of the neural mechanisms of action and clinical efficiency of riluzole in treating amyotrophic lateral sclerosis: what have we learned in the last decade? CNS Neurosci Ther 2011;17(1):4-31

37 Martin D, Thompson MA, Nadler JV. The neuroprotective agent riluzole inhibits release of glutamate and aspartate from slices of hippocampal area CA1. Eur J Pharmacol 1993;250(3):473-476

38 Bensimon G, Lacomblez L, Meininger V; ALS/Riluzole Study Group. A controlled trial of riluzole in amyotrophic lateral sclerosis. N Engl J Med 1994;330(9):585-591

39 Bialer M. Chemical properties of antiepileptic drugs (AEDs). Adv Drug Deliv Rev 2011;(Nov):21

40 Maragakis NJ, Jackson M, Ganel R, Rothstein JD. Topiramate protects against motor neuron degeneration in organotypic spinal cord cultures but not in G93A SOD1 transgenic mice. Neurosci Lett 2003;338(2):107-110

41 Cudkowicz ME, Shefner JM, Schoenfeld DA, et al; Northeast ALS Consortium. A randomized, placebo-controlled trial of topiramate in amyotrophic lateral sclerosis. Neurology 2003;61(4):456-464

42 Kaufmann P, Lomen-Hoerth C. ALS treatment strikes out while trying for a homer: the topiramate trial. Neurology 2003;61 (4):434-435

43 Miller RG, Moore DH II, Gelinas DF, et al; Western ALS Study Group. Phase III randomized trial of gabapentin in patients with amyotrophic lateral sclerosis. Neurology 2001;56(7):843-848

44 Eisen A, Stewart H, Schulzer M, Cameron D. Anti-glutamate therapy in amyotrophic lateral sclerosis: a trial using lamotrigine. Can J Neurol Sci 1993;20(4):297-301

45 Ryberg H, Askmark H, Persson LI. A double-blind randomized clinical trial in amyotrophic lateral sclerosis using lamotrigine: effects on CSF glutamate, aspartate, branched-chain amino acid levels and clinical parameters. Acta Neurol Scand 2003;108(1):1-8

46 Gredal O, Werdelin L, Bak S, et al. A clinical trial of dextromethorphan in amyotrophic lateral sclerosis. Acta Neurol Scand 1997;96 (1):8-13

47 de Carvalho M, Pinto S, Costa J, Evangelista T, Ohana B, Pinto A. A randomized, placebo-controlled trial of memantine for functional disability in amyotrophic lateral sclerosis. Amyotroph Lateral Scler 2010;11(5):456-460

48 Pascuzzi RM, Shefner J, Chappell AS, et al. A phase II trial of talampanel in subjects with amyotrophic lateral sclerosis. Amyotroph Lateral Scler 2010;11(3):266-271

49 Paizs M, Tortarolo M, Bendotti C, Engelhardt JI, Siklós L. Talampanel reduces the level of motoneuronal calcium in transgenic mutant SOD1 mice only if applied presymptomatically. Amyotroph Lateral Scler 2011;12(5):340-344

50 Rothstein JD, Patel S, Regan MR, et al. Beta-lactam antibiotics offer neuroprotection by increasing glutamate transporter expression. Nature 2005;433(7021):73-77

51 U.S. National Institutes of Health. Clinical trial ceftriaxone in subjects with ALS. Available at: http://clinicaltrials.gov/ct2/ show/NCT00349622?term=Ceftriaxone + als\&rank $=1$. Accessed May 22, 2012

52 Ghadge GD, Slusher BS, Bodner A, et al. Glutamate carboxypeptidase II inhibition protects motor neurons from death in familial amyotrophic lateral sclerosis models. Proc Natl Acad Sci U S A 2003;100(16):9554-9559

53 Wechsler IS. The treatment of amyotrophic lateral sclerosis with Vitamin E (tocopherols). Am J Med Sci 1940;200(6):765-778

54 Pascuzzi RM. Blinded and seeing the light, (John Noseworthy), Lou Gehrig and other tales of enlightenment). Semin Neurol 1998;18 (3):415-418 
55 Gurney ME, Cutting FB, Zhai P, et al. Benefit of vitamin E, riluzole, and gabapentin in a transgenic model of familial amyotrophic lateral sclerosis. Ann Neurol 1996;39(2):147-157

56 Desnuelle C, Dib M, Garrel C, Favier A. A double-blind, placebocontrolled randomized clinical trial of alpha-tocopherol (vitamin E) in the treatment of amyotrophic lateral sclerosis. ALS RiluzoleTocopherol Study Group. Amyotroph Lateral Scler Other Motor Neuron Disord 2001;2(1):9-18

57 Graf M, Ecker D, Horowski R, et al; German vitamin E/ALS Study Group. High dose vitamin E therapy in amyotrophic lateral sclerosis as add-on therapy to riluzole: results of a placebo-controlled double-blind study. J Neural Transm 2005;112(5):649-660

58 Wang H, O'Reilly EJ, Weisskopf MG, et al. Vitamin E intake and risk of amyotrophic lateral sclerosis: a pooled analysis of data from 5 prospective cohort studies. Am J Epidemiol 2011;173(6):595-602

59 Andreassen OA, Dedeoglu A, Klivenyi P, Beal MF, Bush AI. N-acetylL-cysteine improves survival and preserves motor performance in an animal model of familial amyotrophic lateral sclerosis. Neuroreport 2000;11(11):2491-2493

60 Orrell RW, Lane RJ, Ross M. A systematic review of antioxidant treatment for amyotrophic lateral sclerosis/motor neuron disease. Amyotroph Lateral Scler 2008;9(4):195-211

61 Pastula DM, Moore DH, Bedlack RS. Creatine for amyotrophic lateral sclerosis/motor neuron disease. Cochrane Database Syst Rev 2010;(6):CD005225

62 Kaneb HM, Sharp PS, Rahmani-Kondori N, Wells DJ. Metformin treatment has no beneficial effect in a dose-response survival study in the SOD1(G93A) mouse model of ALS and is harmful in female mice. PLoS ONE 2011;6(9):e24189

63 Weishaupt JH, Bartels C, Pölking E, et al. Reduced oxidative damage in ALS by high-dose enteral melatonin treatment. J Pineal Res 2006;41(4):313-323

64 Kwieciński H, Janik P, Jamrozik Z, Opuchlik A. [The effect of selegiline and vitamin $E$ in the treatment of ALS: an open randomized clinical trials]. Neurol Neurochir Pol 2001;35(1, Suppl):101-106

65 Cheah BC, Kiernan MC. Dexpramipexole, the R(+) enantiomer of pramipexole, for the potential treatment of amyotrophic lateral sclerosis. IDrugs 2010;13(12):911-920

66 Phase 3 study of dexpramipexole in ALS (EMPOWER). Available at: http://clinicaltrials.gov/ct2/show/NCT01281189?term=Dexpramipexole+als\&rank=3. Accessed May 22, 2012

67 Cudkowicz M, Bozik ME, Ingersoll EW, et al. The effects of dexpramipexole (KNS-760704) in individuals with amyotrophic lateral sclerosis. Nat Med 2011;17(12):1652-1656

68 Baumann J. Results of treatment of certain diseases of the central nervous system with ACTH and corticosteroids. Acta Neurol Scand Suppl 1965;13(Pt 2):453-461

69 Brown RH Jr, Hauser SL, Harrington H, Weiner HL. Failure of immunosuppression with a ten- to 14-day course of high-dose intravenous cyclophosphamide to alter the progression of amyotrophic lateral sclerosis. Arch Neurol 1986;43(4):383-384

70 Monstad I, Dale I, Petlund CF, Sjaastad O. Plasma exchange in motor neuron disease. A controlled study. J Neurol 1979;221(1):59-66

71 Meucci N, Nobile-Orazio E, Scarlato G. Intravenous immunoglobulin therapy in amyotrophic lateral sclerosis. J Neurol 1996;243 (2):117-120

72 Appel SH, Stewart SS, Appel V, et al. A double-blind study of the effectiveness of cyclosporine in amyotrophic lateral sclerosis. Arch Neurol 1988;45(4):381-386

73 Van Den Bosch L, Tilkin P, Lemmens G, Robberecht W. Minocycline delays disease onset and mortality in a transgenic model of ALS. Neuroreport 2002;13(8):1067-1070
74 Gordon PH, Moore DH, Gelinas DF, et al. Placebo-controlled phase I/II studies of minocycline in amyotrophic lateral sclerosis. Neurology 2004;62(10):1845-1847

75 Gordon PH, Moore DH, Miller RG, et al; Western ALS Study Group. Efficacy of minocycline in patients with amyotrophic lateral sclerosis: a phase III randomised trial. Lancet Neurol 2007;6 (12):1045-1053

76 Keller AF, Gravel M, Kriz J. Treatment with minocycline after disease onset alters astrocyte reactivity and increases microgliosis in SOD1 mutant mice. Exp Neurol 2011;228(1):69-79

77 Neuraltus P. Neuraltus Pharmaceuticals reports clinical results from phase 1 NP001 study in the treatment of amyotrophic lateral sclerosis (ALS). Available at: http://www.neuraltus.com/pages/ news_rel11_30_10.html. Accessed May 22, 2012

78 A study of NP001 in subjects with amyotrophic lateral sclerosis (ALS). Available at: http://clinicaltrials.gov/ct2/show/ NCT01281631?term=NP001+als\&rank=2. Accessed May 22, 2012

79 Cytokinetics. Cytokinetics announces company participation and support of ALS fundraising activities in connection with ALS awareness month. Available at: http://www.cytokinetics.com/ press_releases/release/pr_1336516928. Accessed May 22, 2012

80 Shefner J, Cedarbaum JM, Cudkowicz ME, et al. Safety, tolerability and pharmacodynamics of a skeletal muscle activator in amyotrophic lateral sclerosis. Amyotroph Lateral Scler 2012;13(5): 430-438

81 Smith RA, Miller TM, Yamanaka K, et al. Antisense oligonucleotide therapy for neurodegenerative disease. J Clin Invest 2006;116 (8):2290-2296

82 Safety T, and Activity Study of ISIS SOD1Rx to Treat Familial Amyotrophic Lateral Sclerosis (ALS) Caused by SOD1 Gene Mutations (SOD-1). Available at: http://clinicaltrials.gov/ct2/show/ NCT01041222?term=isis+als\&rank=1. Accessed May 22, 2012

83 Wahl M. ISIS-SOD1-Rx: So Far, So Good. MDA/ALS Newsmagazine: MDA ALS division. Available at: http://alsn.mda.org/news/isissod1-rX-so-far-so-good. Accessed April 27, 2011

84 Lanka V, Wieland S, Barber J, Cudkowicz M. Arimoclomol: a potential therapy under development for ALS. Expert Opin Investig Drugs 2009;18(12):1907-1918

85 Phase II/III randomized, placebo-controlled trial of Arimoclomol in SOD1 positive familial amyotrophic lateral sclerosis. Available at: http://clinicaltrials.gov/ct2/show/NCT00706147?term=Arimoclomol+als\&rank=1. Accessed May 22, 2012

86 Broom WJ, Auwarter KE, Ni J, et al. Two approaches to drug discovery in SOD1-mediated ALS. J Biomol Screen 2006;11 (7):729-735

87 Madsen A. Anti-malarial drug takes aim at SOD1. MDA/ALS Newsmagazine. Available at: http://alsn.mda.org/article/anti-malarial-drug-takes-aim-sod1. Accessed September 9, 2009

88 SOD1 inhibition by pyrimethamine in familial amyotrophic lateral sclerosis (ALS). Available at: http://clinicaltrials.gov/ct2/ show/NCT01083667?term=pyrimethamine + als\&rank=1. Accessed May 22, 2012

89 Mazzini L, Mareschi K, Ferrero I, et al. Mesenchymal stromal cell transplantation in amyotrophic lateral sclerosis: a long-term safety study. Cytotherapy 2012;14(1):56-60

90 Karussis D, Karageorgiou C, Vaknin-Dembinsky A, et al. Safety and immunological effects of mesenchymal stem cell transplantation in patients with multiple sclerosis and amyotrophic lateral sclerosis. Arch Neurol 2010;67(10):1187-1194

91 Glass JD, Boulis NM, Johe K, et al. Lumbar intraspinal injection of neural stem cells in patients with ALS: Results of a phase I trial in 12 patients. Stem Cells 2012;(Mar):13 